Interfaces and Free Boundaries 13 (2011), 223-238

DOI $10.4171 / \mathrm{IFB} / 255$

\title{
Free boundary regularity for a problem with right hand side
}

\author{
D. DE SILVA \\ Department of Mathematics, Barnard College, Columbia University, New York, NY 10027, USA \\ E-mail: desilva@math.columbia.edu
}

[Received 17 December 2009 and in revised form 16 March 2011]

\begin{abstract}
We consider a one-phase free boundary problem with variable coefficients and nonzero right hand side. We prove that flat free boundaries are $C^{1, \alpha}$ using a different approach than the classical supconvolution method of Caffarelli. We use this result to deduce that Lipschitz free boundaries are $C^{1, \alpha}$.
\end{abstract}

\section{Introduction}

Consider the following one-phase free boundary problem with variable coefficients and nonzero right hand side:

$$
\begin{cases}\sum_{i, j} a_{i j}(x) u_{i j}=f & \text { in } \Omega^{+}(u):=\{x \in \Omega: u(x)>0\}, \\ |\nabla u|=g & \text { on } F(u):=\partial \Omega^{+}(u) \cap \Omega,\end{cases}
$$

with $\Omega$ a bounded domain in $\mathbb{R}^{n}$, the coefficients $a_{i j} \in C^{0, \beta}(\Omega), f \in C(\Omega) \cap L^{\infty}(\Omega)$, and $g \in C^{0, \beta}(\Omega), g \geqslant 0$.

In this paper we are concerned with the regularity of the set $F(u)$, called the free boundary of $u$. There is an extensive literature on the regularity of the free boundary for this type of problem when $f \equiv 0$. In the case of the Laplace operator, Caffarelli proved in his pioneer work [C1] that Lipschitz free boundaries are $C^{1, \alpha}$, while in [C2] he showed that "flat" free boundaries are Lipschitz. The key step of the method in [C1, C2] consists in finding a family of comparison subsolutions using supconvolutions on balls of variable radii.

Higher regularity of the free boundary follows from the classical work of Kinderlehrer and Nirenberg $[\overline{\mathrm{KN}}]$.

Regularity results in the spirit of [C1, C2] have been subsequently proved for more general operators. In [W1, W2] Wang considered concave fully nonlinear uniformly elliptic operators of the form $\mathcal{F}\left(D^{2} u\right)$. The work [C1] was extended by Feldman [F1, [F2] to a class of nonconcave fully nonlinear uniformly elliptic operators of the type $\mathcal{F}\left(D^{2} u, D u\right)$ and to certain nonisotropic problems. For operators with variable coefficients regularity results are proved in the work of Cerruti, Ferrari and Salsa [CFS], and Ferrari and Salsa [FS1, [FS2]. Also, Ferrari and then Argiolas and Ferrari in $[\mathrm{Fe}, \mathrm{AF}]$ considered a class of fully nonlinear operators of the form $\mathcal{F}\left(D^{2} u, x\right)$ with Hölder dependence on $x$.

The results cited above follow the guidelines of [C1, C2]. One purpose of this paper is to provide a different method of proving that flat free boundaries are $C^{1, \alpha}$. The approach we use is quite flexible since it easily applies to more general nonlinear operators, even degenerate ones, and it also applies to two-phase problems.

In particular, when dealing with operators with variable coefficients we easily deduce that Lipschitz free boundaries are $C^{1, \alpha}$. In fact our flatness result allows us to use a blow-up argument 
and reduce the problem to the case of constant coefficients operators. Our strategy is largely inspired by the work of Savin $[\mathrm{S}]$.

We now state our main results (for the precise definition of viscosity solutions we refer the reader to Section 2). We assume that the matrix $\left(a_{i j}(x)\right)$ is positive definite.

THEOREM 1.1 (Flatness implies $C^{1, \alpha}$ ) Let $u$ be a viscosity solution to (1.1) in $B_{1}$. Assume that $0 \in F(u), g(0)=1$ and $a_{i j}(0)=\delta_{i j}$. There exists a universal constant $\bar{\varepsilon}>0$ such that, if the graph of $u$ is $\bar{\varepsilon}$-flat in $B_{1}$, i.e.

$$
\left(x_{n}-\bar{\varepsilon}\right)^{+} \leqslant u(x) \leqslant\left(x_{n}+\bar{\varepsilon}\right)^{+}, \quad x \in B_{1},
$$

and

$$
\left[a_{i j}\right]_{C^{0, \beta}\left(B_{1}\right)} \leqslant \bar{\varepsilon}, \quad\|f\|_{L^{\infty}\left(B_{1}\right)} \leqslant \bar{\varepsilon}, \quad[g]_{C^{0, \beta}\left(B_{1}\right)} \leqslant \bar{\varepsilon}
$$

then $F(u)$ is $C^{1, \alpha}$ in $B_{1 / 2}$.

THEOREM 1.2 (Lipschitz implies $C^{1, \alpha}$ ) Let $u$ be a viscosity solution to 1.1 . Assume that $0 \in$ $F(u)$ and $g(0)>0$. If $F(u)$ is a Lipschitz graph in a neighborhood of 0 , then $F(u)$ is $C^{1, \alpha}$ in a (smaller) neighborhood of 0 .

In the theorem above, the size of the neighborhood where $F(u)$ is $C^{1, \alpha}$ depends on the radius $\rho$ of the ball $B_{\rho}$ where $F(u)$ is Lipschitz, on the Lipschitz norm of $F(u)$, on $\left[a_{i j}\right]_{C^{0, \beta}\left(B_{\rho}\right)},\|g\|_{C^{0, \beta}\left(B_{\rho}\right)}$, and $\|f\|_{L^{\infty}\left(B_{\rho}\right)}$.

We remark that the assumptions on the coefficients $a_{i j}(x)$ in Theorem 1.1 can be weakened to a Cordes-Nirenberg type condition

$$
\left\|a_{i j}-\delta_{i j}\right\|_{L^{\infty}\left(B_{1}\right)} \leqslant \delta(n) .
$$

As already pointed out, our strategy of the proof of Theorem 1.1 is inspired by $[\mathrm{S}]$. The main idea is to show that the graph of $u$ enjoys an "improvement of flatness" property, that is, if the graph of $u$ oscillates $\varepsilon$ away from a hyperplane in $B_{1}$, then in $B_{r_{0}}$ it oscillates $\varepsilon r_{0} / 2$ away from possibly a different hyperplane. The key tool in proving this property will be a Harnack type inequality for solutions to a one-phase free boundary problem.

Theorem 1.2 will follow via a blow-up argument from Theorem 1.1 and the classical theory in [C1].

The problem (1.1), in which a right hand side appears, is not specifically dealt with in any of the previous cited works. Our interest in this problem arises in connection with the question of the regularity of the free surface which occurs in the classical hydrodynamical problem for traveling two-dimensional gravity water-waves with vorticity. There has been considerable interest in this problem in recent years, starting with the systematic study of Constantin and Strauss [CS].

The physical situation is the following: a traveling wave of an incompressible, inviscid, heavy fluid moves with constant speed over a horizontal surface. Since the fluid is incompressible, the flow can be described by a stream function $u$ which solves the following free boundary problem (in 2D):

$$
\begin{aligned}
& \Delta u=-\gamma(u) \quad \text { in } \Omega:=\left\{(x, y) \in \mathbb{R}^{2}: 0<u(x, y)<B\right\}, \\
& u=B \quad \text { on } y=0, \\
& |\nabla u|^{2}+2 g y=Q \quad \text { on } S:=\{u=0\},
\end{aligned}
$$

with $B, g$ fixed constants, $\gamma$ a given vorticity function and $Q$ a parameter. Of special interest are those free boundaries which are given by the graph of a function $y=\psi(x)$. In the regions where $\psi$ 
is decreasing (resp. increasing) the free boundary is Lipschitz with respect to the direction $e_{1}+e_{2}$ (resp. $e_{2}-e_{1}$ ) and moreover $Q-2 g y>0$. As a consequence of Theorem 1.2, the free boundary is smooth in these regions.

The free boundary is not expected to be smooth at the so-called stagnation points where $Q=2 g y$. At such points, the profile of an irrotational wave $(\gamma \equiv 0)$ has a corner with included angle of $120^{\circ}$. This was conjectured by Stokes and it was proved by Amick, Fraenkel, and Toland [AFT], and by Plotnikov $[\mathrm{P}]$. The case $\gamma \neq 0$ was investigated by Varvaruca in $[\mathrm{V}]$ and recently by Varvaruca and Weiss in [VW].

The paper is organized as follows. In Section 2 we introduce notation and definitions and we prove a regularity result for viscosity solutions to a Neumann problem which we will use in the proof of Theorem 1.1 Next, in Section 3, we present the statement of our Harnack inequality and we exhibit its proof. In Section 4, we state and prove the "improvement of flatness" lemma. Finally, in Section 5, we provide the proofs of Theorems 1.1 and 1.2. We conclude the paper with an Appendix in which we prove the standard Lipschitz continuity and nondegeneracy of solutions to a one-phase free boundary problem.

\section{Preliminaries}

In this section we provide notation and definitions used throughout the paper. We also present an auxiliary result which will be used in the proof of our main Theorem 1.1 .

Notation. For any continuous function $u: \Omega \subset \mathbb{R}^{n} \rightarrow \mathbb{R}$ we denote

$$
\Omega^{+}(u):=\{x \in \Omega: u(x)>0\}, \quad F(u):=\partial \Omega^{+}(u) \cap \Omega .
$$

We refer to the set $F(u)$ as the free boundary of $u$, while $\Omega^{+}(u)$ is its positive phase (or side).

We now state the definition of viscosity solution to the problem under consideration, that is,

$$
\begin{cases}\sum_{i, j} a_{i j}(x) u_{i j}=f & \text { in } \Omega^{+}(u), \\ |\nabla u|=g & \text { on } F(u) .\end{cases}
$$

Here $\Omega$ is a bounded domain in $\mathbb{R}^{n}, a_{i j} \in C^{0, \beta}(\Omega), f \in C(\Omega) \cap L^{\infty}(\Omega), g \in C^{0, \beta}(\Omega)$, and $g \geqslant 0$.

First we need the following standard notion.

DEFINITION 2.1 Given $u, \varphi \in C(\Omega)$, we say that $\varphi$ touches $u$ from below (resp. above) at $x_{0} \in \Omega$ if $u\left(x_{0}\right)=\varphi\left(x_{0}\right)$ and

$$
u(x) \geqslant \varphi(x) \quad(\text { resp. } u(x) \leqslant \varphi(x)) \quad \text { in a neighborhood } O \text { of } x_{0} .
$$

If this inequality is strict in $O \backslash\left\{x_{0}\right\}$, we say that $\varphi$ touches $u$ strictly from below (resp. above).

Definition 2.2 Let $u$ be a nonnegative continuous function in $\Omega$. We say that $u$ is a viscosity solution to 2.1 in $\Omega$ if the following conditions are satisfied:

(i) $\sum_{i, j} a_{i j}(x) u_{i j}=f$ in $\Omega^{+}(u)$ in the viscosity sense, i.e. if $\varphi \in C^{2}\left(\Omega^{+}(u)\right)$ touches $u$ from below (resp. above) at $x_{0} \in \Omega^{+}(u)$ then

$$
\sum_{i, j} a_{i j}\left(x_{0}\right) \varphi_{i j}\left(x_{0}\right) \leqslant f\left(x_{0}\right) \quad\left(\text { resp. } \sum_{i, j} a_{i j}\left(x_{0}\right) \varphi_{i j}\left(x_{0}\right) \geqslant f\left(x_{0}\right)\right) .
$$


(ii) If $\varphi \in C^{2}(\Omega)$ and $\varphi^{+}$touches $u$ from below (resp. above) at $x_{0} \in F(u)$ and $|\nabla \varphi|\left(x_{0}\right) \neq 0$ then

$$
|\nabla \varphi|\left(x_{0}\right) \leqslant g\left(x_{0}\right) \quad\left(\text { resp. }|\nabla \varphi|\left(x_{0}\right) \geqslant g\left(x_{0}\right)\right) .
$$

Viscosity solutions are introduced to be able to use comparison techniques. To this end, we will need the following notion of comparison subsolution/supersolution.

DEFINITION 2.3 Let $v \in C^{2}(\Omega)$. We say that $v$ is a strict (comparison) subsolution (resp. supersolution) to 2.1 in $\Omega$ if the following conditions are satisfied:

(i) $\sum_{i, j} a_{i j}(x) v_{i j}>f(x)$ (resp. $\left.<f(x)\right)$ in $\Omega^{+}(v)$.

(ii) If $x_{0} \in F(v)$, then

$$
|\nabla v|\left(x_{0}\right)>g\left(x_{0}\right) \quad\left(\text { resp. } 0<|\nabla v|\left(x_{0}\right)<g\left(x_{0}\right)\right) .
$$

Notice that, by the implicit function theorem, if $v$ is a strict subsolution/supersolution then $F(v)$ is a $C^{2}$ hypersurface.

The following lemma is an immediate consequence of the definitions above.

LEMMA 2.4 Let $u, v$ be respectively a solution and a strict subsolution to 2.1 in $\Omega$. If $u \geqslant v^{+}$ in $\Omega$ then $u>v^{+}$in $\Omega^{+}(v) \cup F(v)$.

Notation. Hereafter $B_{\rho}\left(x_{0}\right) \subset \mathbb{R}^{n}$ denotes the open ball of radius $\rho$ centered at $x_{0}$, and $B_{\rho}=$ $B_{\rho}(0)$. A positive constant depending only on the dimension $n$ is called a universal constant. We often use $c, c_{i}$ to denote small universal constants, and $C, C_{i}$ to denote large universal constants.

Our main Theorem 1.1 will follow from the regularity properties of solutions to the classical Neumann problem for the Laplace operator. Precisely, we consider the following boundary value problem:

$$
\begin{cases}\Delta \tilde{u}=0 & \text { in } B_{\rho} \cap\left\{x_{n}>0\right\}, \\ \tilde{u}_{n}=0 & \text { on } B_{\rho} \cap\left\{x_{n}=0\right\} .\end{cases}
$$

We use the notion of viscosity solution to 2.2). For completeness (and for lack of references), we recall standard notions and we prove regularity of viscosity solutions.

DEFINITION 2.5 Let $\tilde{u}$ be a continuous function on $B_{\rho} \cap\left\{x_{n} \geqslant 0\right\}$. We say that $\tilde{u}$ is a viscosity solution to 2.2) if given a quadratic polynomial $P(x)$ touching $\tilde{u}$ from below (resp. above) at $\bar{x} \in B_{\rho} \cap\left\{x_{n} \geqslant 0\right\}$,

(i) if $\bar{x} \in B_{\rho} \cap\left\{x_{n}>0\right\}$ then $\Delta P \leqslant 0$ (resp. $\Delta P \geqslant 0$ ), i.e. $\tilde{u}$ is harmonic in the viscosity sense;

(ii) if $\bar{x} \in B_{\rho} \cap\left\{x_{n}=0\right\}$ then $P_{n}(\bar{x}) \leqslant 0$ (resp. $\left.P_{n}(\bar{x}) \geqslant 0\right)$.

REMARK Notice that in the definition above we can choose polynomials $P$ that touch $\tilde{u}$ strictly from below/above (replace $P$ by $P_{\eta}(x)=P(x)-\eta\left(x_{n}-\bar{x}_{n}\right)^{2}$ and then let $\eta$ go to 0 ).

Also, it suffices to verify that (ii) holds for polynomials $\tilde{P}$ with $\Delta \tilde{P}>0$. Indeed, let $P$ touch $\tilde{u}$ from below at $\bar{x}$. Then

$$
\tilde{P}=P-\eta\left(x_{n}-\bar{x}_{n}\right)+C(\eta)\left(x_{n}-\bar{x}_{n}\right)^{2}
$$

touches $\tilde{u}$ from below at $\bar{x}$ (for a sufficiently small constant $\eta>0$ and a large constant $C>0$ depending on $\eta$ ) and satisfies

$$
\Delta \tilde{P}>0, \quad \tilde{P}_{n}(\bar{x})=P_{n}(\bar{x})-\eta
$$


If (ii) holds for strictly subharmonic polynomials, we get $\tilde{P}_{n}(\bar{x}) \leqslant \eta$, which by letting $\eta$ go to 0 implies $P_{n}(\bar{x}) \leqslant 0$.

Lemma 2.6 Let $\tilde{u}$ be a viscosity solution to 2.2. Then $\tilde{u}$ is a classical solution to 2.2. In particular, $\tilde{u} \in C^{\infty}\left(B_{\rho} \cap\left\{x_{n} \geqslant 0\right\}\right)$.

Proof. Let

$$
u^{*}(x)= \begin{cases}\tilde{u}(x) & \text { if } x \in B_{\rho} \cap\left\{x_{n} \geqslant 0\right\} \\ \tilde{u}\left(x^{\prime},-x_{n}\right) & \text { if } x \in B_{\rho} \cap\left\{x_{n}<0\right\}\end{cases}
$$

where $x^{\prime}=\left(x_{1}, \ldots, x_{n-1}\right)$.

We claim that $u^{*}$ is harmonic (in the viscosity sense), and hence smooth, in $B_{\rho}$. Indeed, let $P$ be a polynomial touching $u^{*}$ at $\bar{x} \in B_{\rho}$ strictly from below. We need to show that $\Delta P \leqslant 0$. Clearly, we only need to consider the case when $\bar{x} \in\left\{x_{n}=0\right\}$.

Consider the polynomial

$$
S(x)=\frac{P(x)+P\left(x^{\prime},-x_{n}\right)}{2} .
$$

Then

$$
\Delta S=\Delta P, \quad S_{n}\left(x^{\prime}, 0\right)=0 .
$$

Also, $S$ still touches $u^{*}$ strictly from below at $\bar{x}$. Now, consider the family of polynomials

$$
S_{\varepsilon}=S+\varepsilon x_{n}, \quad \varepsilon>0 .
$$

For $\varepsilon$ small $S_{\varepsilon}$ will touch $u^{*}$ from below at some point $x_{\varepsilon}$.

If $x_{\varepsilon}$ belongs to $\left\{x_{n}=0\right\}$, since $S_{\varepsilon}$ touches $\tilde{u}$ from below at $x_{\varepsilon}$ and $\tilde{u}_{n}\left(x^{\prime}, 0\right)=0$ in the viscosity sense, we obtain

$$
\left(S_{\varepsilon}\right)_{n}\left(x_{\varepsilon}^{\prime}, 0\right) \leqslant 0, \quad \text { i.e. } \quad S_{n}\left(x_{\varepsilon}^{\prime}, 0\right)+\varepsilon \leqslant 0,
$$

contradicting 2.3).

Thus $x_{\varepsilon} \in B_{\rho} \backslash\left\{x_{n}=0\right\}$ and hence $\Delta S=\Delta P \leqslant 0$.

In conclusion, $u^{*}$ is harmonic in $B_{\rho}$ and our statement immediately follows.

\section{A Harnack inequality}

In this section we will prove a Harnack type inequality for a solution $u$ to our problem

$$
\begin{cases}\sum_{i, j} a_{i j}(x) u_{i j}=f & \text { in } \Omega^{+}(u), \\ |\nabla u|=g & \text { on } F(u),\end{cases}
$$

under the assumption $(0<\varepsilon<1)$

$$
\|f\|_{L^{\infty}(\Omega)} \leqslant \varepsilon^{2}, \quad\|g-1\|_{L^{\infty}(\Omega)} \leqslant \varepsilon^{2}, \quad\left\|a_{i j}-\delta_{i j}\right\|_{L^{\infty}(\Omega)} \leqslant \varepsilon .
$$

This theorem roughly says that if the graph of $u$ oscillates $\varepsilon r$ away from $x_{n}^{+}$in $B_{r}$, then it oscillates $(1-c) \varepsilon r$ in $B_{r / 20}$. A corollary of this theorem will be a key tool in the proof of Theorem 1.1 . 
THEOREM 3.1 (Harnack inequality) There exists a universal constant $\bar{\varepsilon}$ such that if $u$ solves (3.1)(3.2), and for some point $x_{0} \in \Omega^{+}(u) \cup F(u)$,

$$
\left(x_{n}+a_{0}\right)^{+} \leqslant u(x) \leqslant\left(x_{n}+b_{0}\right)^{+} \quad \text { in } B_{r}\left(x_{0}\right) \subset \Omega
$$

with

$$
b_{0}-a_{0} \leqslant \varepsilon r, \quad \varepsilon \leqslant \bar{\varepsilon},
$$

then

$$
\left(x_{n}+a_{1}\right)^{+} \leqslant u(x) \leqslant\left(x_{n}+b_{1}\right)^{+} \quad \text { in } B_{r / 20}\left(x_{0}\right)
$$

with

$$
a_{0} \leqslant a_{1} \leqslant b_{1} \leqslant b_{0}, \quad b_{1}-a_{1} \leqslant(1-c) \varepsilon r,
$$

and $0<c<1$ universal.

From this statement we immediately get the desired corollary to be used in the proof of our main result. Precisely, if $u$ satisfies 3.3 with $r=1$, then we can apply the Harnack inequality repeatedly to obtain

$$
\left(x_{n}+a_{m}\right)^{+} \leqslant u(x) \leqslant\left(x_{n}+b_{m}\right)^{+} \quad \text { in } B_{20^{-m}}\left(x_{0}\right)
$$

with

$$
b_{m}-a_{m} \leqslant(1-c)^{m} \varepsilon
$$

for all $m$ 's such that

$$
(1-c)^{m} 20^{m} \varepsilon \leqslant \bar{\varepsilon}
$$

This implies that for all such $m$ 's, the oscillation of the function

$$
\tilde{u}_{\varepsilon}(x)=\frac{u(x)-x_{n}}{\varepsilon}
$$

in $\left(\Omega^{+}(u) \cup F(u)\right) \cap B_{r}\left(x_{0}\right), r=20^{-m}$, is less than $(1-c)^{m}=20^{-\gamma m}=r^{\gamma}$. Thus, the following corollary holds.

Corollary 3.2 Let $u$ be a solution to (3.1)- 3.2 satisfying 3.3 for $r=1$. Then in $B_{1}\left(x_{0}\right)$, $\tilde{u}_{\varepsilon}$ has a Hölder modulus of continuity at $x_{0}$, outside the ball of radius $\varepsilon / \bar{\varepsilon}$, i.e. for all $x \in\left(\Omega^{+}(u) \cup\right.$ $F(u)) \cap B_{1}\left(x_{0}\right)$ with $\left|x-x_{0}\right| \geqslant \varepsilon / \bar{\varepsilon}$,

$$
\left|\tilde{u}_{\varepsilon}(x)-\tilde{u}_{\varepsilon}\left(x_{0}\right)\right| \leqslant C\left|x-x_{0}\right|^{\gamma} .
$$

The proof of the Harnack inequality relies on the following lemma.

LEMmA 3.3 There exists a universal constant $\bar{\varepsilon}>0$ such that if $u$ is a solution to 3.1 -3.2 in $B_{1}$ with $0<\varepsilon \leqslant \bar{\varepsilon}$ and $u$ satisfies

$$
p(x)^{+} \leqslant u(x) \leqslant(p(x)+\varepsilon)^{+}, \quad x \in B_{1}, p(x)=x_{n}+\sigma,|\sigma|<1 / 10,
$$

then if at $\bar{x}=\frac{1}{5} e_{n}$,

$$
u(\bar{x}) \geqslant(p(\bar{x})+\varepsilon / 2)^{+},
$$

then

$$
u \geqslant(p+c \varepsilon)^{+} \quad \text { in } \bar{B}_{1 / 2}
$$


for some $0<c<1$. Analogously, if

$$
u(\bar{x}) \leqslant(p(\bar{x})+\varepsilon / 2)^{+},
$$

then

$$
u \leqslant(p+(1-c) \varepsilon)^{+} \quad \text { in } \bar{B}_{1 / 2} .
$$

Proof. We prove the first statement. Clearly, from 3.4],

$$
u \geqslant p \quad \text { in } B_{1} .
$$

Let

$$
w=c\left(|x-\bar{x}|^{-\gamma}-(3 / 4)^{-\gamma}\right)
$$

be defined in the closure of the annulus

$$
A:=B_{3 / 4}(\bar{x}) \backslash \bar{B}_{1 / 20}(\bar{x}) .
$$

The constant $c$ is such that $w$ satisfies the boundary conditions

$$
\begin{cases}w=0 & \text { on } \partial B_{3 / 4}(\bar{x}) \\ w=1 & \text { on } \partial B_{1 / 20}(\bar{x})\end{cases}
$$

Also, since $\left\|a_{i j}-\delta_{i j}\right\|_{L^{\infty}\left(B_{1}\right)} \leqslant \varepsilon$ the matrix $\left(a_{i j}\right)$ is uniformly elliptic and we can choose the constant $\gamma$ universal so that

$$
\sum_{i, j} a_{i j}(x) w_{i j} \geqslant \delta>0 \quad \text { in } A,
$$

with $\delta$ universal. Extend $w$ to be equal to 1 on $B_{1 / 20}(\bar{x})$.

Notice that since $|\sigma|<1 / 10$ using 3.7] we get

$$
B_{1 / 10}(\bar{x}) \subset B_{1}^{+}(u) .
$$

Also,

$$
B_{1 / 2} \subset \subset B_{3 / 4}(\bar{x}) \subset \subset B_{1} .
$$

Since in view of 3.7$]-3.8, u-p \geqslant 0$ and solves a uniformly elliptic equation in $B_{1 / 10}(\bar{x})$ with right-hand side $f$, we can apply the Harnack inequality to obtain

$$
u(x)-p(x) \geqslant c(u(\bar{x})-p(\bar{x}))-C\|f\|_{L^{\infty}} \quad \text { in } \bar{B}_{1 / 20}(\bar{x}) .
$$

From 3.5 and the first inequality in 3.2 we conclude that (for $\varepsilon$ small enough)

$$
u-p \geqslant c \varepsilon-C \varepsilon^{2} \geqslant c_{0} \varepsilon \quad \text { in } \bar{B}_{1 / 20}(\bar{x}) .
$$

Now set

$$
v(x)=p(x)+c_{0} \varepsilon(w(x)-1), \quad x \in \bar{B}_{3 / 4}(\bar{x}),
$$

and for $t \geqslant 0$,

$$
v_{t}(x)=v(x)+t, \quad x \in \bar{B}_{3 / 4}(\bar{x}) .
$$


Notice that

$$
\sum_{i j} a_{i j}(x)\left(v_{t}\right)_{i j} \geqslant c_{0} \delta \varepsilon>\varepsilon^{2} \quad \text { in } A .
$$

According to 3.7 and the definition of $v_{t}$ we have

$$
v_{0}(x)=v(x) \leqslant p(x) \leqslant u(x), \quad x \in \bar{B}_{3 / 4}(\bar{x}) .
$$

Let $\bar{t}$ be the largest $t \geqslant 0$ such that

$$
v_{t}(x) \leqslant u(x) \quad \text { in } \bar{B}_{3 / 4}(\bar{x}) .
$$

We want to show that $\bar{t} \geqslant c_{0} \varepsilon$. Then, using the definition 3.11) of $v(x)$ we get

$$
u(x) \geqslant v(x)+\bar{t} \geqslant p(x)+c_{0} \varepsilon w(x)
$$

and hence, since on $\bar{B}_{1 / 2} \subset B_{3 / 4}(\bar{x})$ one has $w(x) \geqslant c_{2}$ for some universal constant $c_{2}$, we obtain

$$
u(x)-p(x) \geqslant c \varepsilon \quad \text { on } \bar{B}_{1 / 2}
$$

as desired.

Suppose $\bar{t}<c_{0} \varepsilon$. Then at some $\tilde{x} \in \bar{B}_{3 / 4}(\bar{x})$ we have

$$
v_{\bar{t}}(\tilde{x})=u(\tilde{x}) .
$$

We show that such a touching point can only occur on $\bar{B}_{1 / 20}(\bar{x})$. Indeed, since $w \equiv 0$ on $\partial B_{3 / 4}(\bar{x})$, from the definition of $v_{t}$ we get

$$
v_{\bar{t}}(x)=p(x)-c_{0} \varepsilon+\bar{t} \quad \text { on } \partial B_{3 / 4}(\bar{x}) .
$$

Using that $\bar{t}<c_{0} \varepsilon$ together with the fact that $u \geqslant p$ we then obtain

$$
v_{\bar{t}}<u \quad \text { on } \partial B_{3 / 4}(\bar{x})
$$

We now show that $\tilde{x}$ cannot belong to the annulus $A$. As already observed,

$$
\sum_{i j} a_{i j}(x)\left(v_{\bar{t}}\right)_{i j}>\varepsilon^{2} \quad \text { in } A
$$

and also

$$
\left|\nabla v_{\bar{t}}\right| \geqslant\left|v_{n}\right|=\left|1+c_{0} \varepsilon w_{n}\right| \quad \text { in } A
$$

We claim that

$$
w_{n}(x) \geqslant c_{1} \quad \text { on }\left\{v_{\bar{t}} \leqslant 0\right\} \cap A,
$$

for a universal constant $c_{1}$.

Indeed, since $w$ is radially symmetric,

$$
w_{n}(x)=|\nabla w(x)| v_{x} \cdot e_{n}, \quad x \in A,
$$


where $v_{x}$ is the unit direction of $x-\bar{x}$. Clearly from the formula for $w$ we get $|\nabla w|>c$ on $A$. Also, $v_{x} \cdot e_{n}$ is bounded below in the region $\left\{v_{\bar{t}} \leqslant 0\right\} \cap A$, since for $\varepsilon$ small enough,

$$
\left\{v_{\bar{t}} \leqslant 0\right\} \cap A \subset\left\{p \leqslant c_{0} \varepsilon\right\}=\left\{x_{n} \leqslant-\sigma+c_{0} \varepsilon\right\} \subset\left\{x_{n}<3 / 20\right\},
$$

and $\bar{x}=\frac{1}{5} e_{n}$.

Hence, from 3.12 we deduce that

$$
\left|\nabla v_{\bar{t}}\right| \geqslant 1+c_{2} \varepsilon \quad \text { on }\left\{v_{\bar{t}} \leqslant 0\right\} \cap A .
$$

In particular, for $\varepsilon$ small enough and in view of the second inequality in 3.2 ,

$$
\left|\nabla v_{\bar{t}}\right|(x)>1+\varepsilon^{2} \geqslant g(x) \quad \text { for } x \in A \cap F\left(v_{\bar{t}}\right) .
$$

Thus, $v_{\bar{t}}$ is a strict subsolution to 3.1 in $A$ and according to Lemma 2.4 since $u$ solves $(3.1)$ in $B_{1}$, $\tilde{x}$ cannot belong to $A$. Therefore, $\tilde{x} \in \bar{B}_{1 / 20}(\bar{x})$ and

$$
u(\tilde{x})=v_{\bar{t}}(\tilde{x}) \leqslant p(\tilde{x})+\bar{t}<p(\tilde{x})+c_{0} \varepsilon,
$$

which implies

$$
u(\tilde{x})-p(\tilde{x})<c_{0} \varepsilon
$$

contradicting 3.10 .

The second statement follows by a similar argument.

We are now ready to give the proof of the Harnack inequality.

Proof of Theorem 3.1 Assume without loss of generality

$$
x_{0}=0, \quad r=1 .
$$

According to 3.3 ,

$$
p(x)^{+} \leqslant u(x) \leqslant(p(x)+\varepsilon)^{+} \quad \text { in } B_{1}
$$

with $p(x)=x_{n}+a_{0}$. If $\left|a_{0}\right|<1 / 10$ then we can apply the previous Lemma 3.3. and the desired statement immediately follows.

Now suppose otherwise. If $a_{0}<-1 / 10$, then (for $\varepsilon$ small) 0 belongs to the zero phase of $(p(x)+\varepsilon)^{+}$, which implies that 0 also belongs to the zero phase of $u$, a contradiction.

If $a_{0}>1 / 10$ then $B_{1 / 10} \subset B_{1}^{+}(u)$, and the conclusion follows by the classical Harnack inequality in $B_{1 / 10}$ as long as $\varepsilon$ is small enough.

\section{Improvement of flatness}

In this section we present the main "improvement of flatness" lemma, from which Theorem 1.1 will easily follow via an iterative argument.

LEMMA 4.1 (Improvement of flatness) Let $u$ be a solution to 3.1 - 3.2 in $B_{1}$ satisfying

$$
\left(x_{n}-\varepsilon\right)^{+} \leqslant u(x) \leqslant\left(x_{n}+\varepsilon\right)^{+} \quad \text { for } x \in B_{1},
$$

and with $0 \in F(u)$. If $0<r \leqslant r_{0}$ for $r_{0}$ a universal constant and $0<\varepsilon \leqslant \varepsilon_{0}$ for some $\varepsilon_{0}$ depending on $r$, then

$$
(x \cdot v-r \varepsilon / 2)^{+} \leqslant u(x) \leqslant(x \cdot v+r \varepsilon / 2)^{+} \quad \text { for } x \in B_{r},
$$

with $|\nu|=1$ and $\left|\nu-e_{n}\right| \leqslant C \varepsilon^{2}$ for a universal constant $C$. 
Proof. We divide the proof into three steps. We use the following notation:

$$
\Omega_{\rho}(u):=\left(B_{1}^{+}(u) \cup F(u)\right) \cap B_{\rho} .
$$

Step 1: Compactness. Fix $r \leqslant r_{0}$ with $r_{0}$ universal (the precise $r_{0}$ will be given in Step 3). Assume for contradiction that we can find a sequence $\varepsilon_{k} \rightarrow 0$ and a sequence $u_{k}$ of solutions to (3.1) in $B_{1}$ with coefficients $a_{i j}^{k}$, right hand side $f_{k}$ and free boundary condition $g_{k}$ satisfying (3.2), such that $u_{k}$ satisfies 4.1, i.e.

$$
\left(x_{n}-\varepsilon_{k}\right)^{+} \leqslant u_{k}(x) \leqslant\left(x_{n}+\varepsilon_{k}\right)^{+} \quad \text { for } x \in B_{1}, 0 \in F\left(u_{k}\right),
$$

but it does not satisfy the conclusion 4.2 of the lemma.

Set

$$
\tilde{u}_{k}(x)=\frac{u_{k}(x)-x_{n}}{\varepsilon_{k}}, \quad x \in \Omega_{1}\left(u_{k}\right) .
$$

Then 4.3 gives

$$
-1 \leqslant \tilde{u}_{k}(x) \leqslant 1 \quad \text { for } x \in \Omega_{1}\left(u_{k}\right) .
$$

From Corollary 3.2, it follows that the function $\tilde{u}_{k}$ satisfies

$$
\left|\tilde{u}_{k}(x)-\tilde{u}_{k}(y)\right| \leqslant C|x-y|^{\gamma}
$$

for $C$ universal and

$$
|x-y| \geqslant \varepsilon_{k} / \bar{\varepsilon}, \quad x, y \in \Omega_{1 / 2}\left(u_{k}\right) .
$$

From 4.3 it clearly follows that $F\left(u_{k}\right)$ converges to $B_{1} \cap\left\{x_{n}=0\right\}$ in the Hausdorff distance. This fact and (4.5) together with Ascoli-Arzelà give that as $\varepsilon_{k} \rightarrow 0$ the graphs of the $\tilde{u}_{k}$ over $\Omega_{1 / 2}\left(u_{k}\right)$ converge (up to a subsequence) in the Hausdorff distance to the graph of a Hölder continuous function $\tilde{u}$ over $B_{1 / 2} \cap\left\{x_{n} \geqslant 0\right\}$.

Step 2: Limiting solution. We now show that $\tilde{u}$ solves

$$
\begin{cases}\Delta \tilde{u}=0 & \text { in } B_{1 / 2} \cap\left\{x_{n}>0\right\}, \\ \tilde{u}_{n}=0 & \text { on } B_{1 / 2} \cap\left\{x_{n}=0\right\},\end{cases}
$$

in the sense of Definition 2.5

Let $P(x)$ be a quadratic polynomial touching $\tilde{u}$ at $\bar{x} \in B_{1 / 2} \cap\left\{x_{n} \geqslant 0\right\}$ strictly from below. We need to show that

(i) if $\bar{x} \in B_{1 / 2} \cap\left\{x_{n}>0\right\}$ then $\Delta P(\bar{x}) \leqslant 0$;

(ii) if $\bar{x} \in B_{1 / 2} \cap\left\{x_{n}=0\right\}$ then $P_{n}(\bar{x}) \leqslant 0$.

Since $\tilde{u}_{k} \rightarrow \tilde{u}$ in the sense specified above, there exist points $x_{k} \in \Omega_{1 / 2}\left(u_{k}\right), x_{k} \rightarrow \bar{x}$, and constants $c_{k} \rightarrow 0$ such that

$$
P\left(x_{k}\right)+c_{k}=\tilde{u}_{k}\left(x_{k}\right)
$$

and

$$
\tilde{u}_{k} \geqslant P+c_{k} \quad \text { in a neighborhood of } x_{k} .
$$

From the definition of $\tilde{u}_{k}$, 4.7 and 4.8 read

$$
u_{k}\left(x_{k}\right)=Q\left(x_{k}\right)
$$


and

$$
u_{k}(x) \geqslant Q(x) \quad \text { in a neighborhood of } x_{k}
$$

where

$$
Q(x)=\varepsilon_{k}\left(P(x)+c_{k}\right)+x_{n} .
$$

We now distinguish two cases.

(i) If $\bar{x} \in B_{1 / 2} \cap\left\{x_{n}>0\right\}$ then $x_{k} \in B_{1 / 2}^{+}\left(u_{k}\right)$ (for $k$ large) and hence, since $Q$ touches $u_{k}$ from below at $x_{k}$, we get

$$
\sum_{i, j} a_{i j}^{k}\left(x_{k}\right) Q_{i j}=\varepsilon_{k} \sum_{i, j} a_{i j}^{k}\left(x_{k}\right) P_{i j} \leqslant f_{k}\left(x_{k}\right) \leqslant \varepsilon_{k}^{2} .
$$

Thus, in view of the last inequality in 3.2.,

$$
\Delta P=\sum_{i, j}\left(\delta_{i j}-a_{i j}^{k}\left(x_{k}\right)\right) P_{i j}+\sum_{i, j} a_{i j}^{k}\left(x_{k}\right) P_{i j} \leqslant C \varepsilon_{k} .
$$

Passing to the limit as $k \rightarrow+\infty$ we conclude that $\Delta P \leqslant 0$ as desired.

(ii) If $\bar{x} \in B_{1 / 2} \cap\left\{x_{n}=0\right\}$, as observed in the Remark following Definition 2.5. we can assume that $\Delta P>0$. We claim that for $k$ large enough, $x_{k} \in F\left(u_{k}\right)$. Otherwise $x_{k_{n}} \in B_{1}^{+}\left(u_{k_{n}}\right)$ for a subsequence $k_{n} \rightarrow \infty$ and as in case (i),

$$
\Delta P \leqslant C \varepsilon_{k_{n}} .
$$

Letting $k_{n} \rightarrow \infty$ we contradict the fact that $P$ is strictly subharmonic. Thus $x_{k} \in F\left(u_{k}\right)$ for $k$ large. Now notice that

$$
\nabla Q=\varepsilon_{k} \nabla P+e_{n},
$$

thus, for $k$ large, $|\nabla Q|>0$. Since $Q^{+}$touches $u_{k}$ from below,

$$
|\nabla Q|\left(x_{k}\right) \leqslant g_{k}\left(x_{k}\right) \leqslant 1+\varepsilon_{k}^{2},
$$

which gives

$$
|\nabla Q|^{2}\left(x_{k}\right)=\varepsilon_{k}^{2}|\nabla P|^{2}\left(x_{k}\right)+1+2 \varepsilon_{k} P_{n}\left(x_{k}\right) \leqslant 1+3 \varepsilon_{k}^{2},
$$

and thus (after division by $\varepsilon_{k}$ )

$$
\varepsilon_{k}|\nabla P|^{2}\left(x_{k}\right)-3 \varepsilon_{k}+2 P_{n}\left(x_{k}\right) \leqslant 0 .
$$

Passing to the limit as $k \rightarrow+\infty$ we obtain $P_{n}(\bar{x}) \leqslant 0$ as desired.

Step 3: Improvement of flatness. From the previous step, $\tilde{u}$ solves (4.6) and from 4.4,

$$
-1 \leqslant \tilde{u} \leqslant 1 \quad \text { in } B_{1 / 2} \cap\left\{x_{n} \geqslant 0\right\} .
$$

From Lemma 2.6 and the bound above we find that, for the given $r$,

$$
|\tilde{u}(x)-\tilde{u}(0)-\nabla \tilde{u}(0) \cdot x| \leqslant C_{0} r^{2} \quad \text { in } B_{r} \cap\left\{x_{n} \geqslant 0\right\},
$$

for a universal constant $C_{0}$. In particular, since $0 \in F(\tilde{u})$ and also $\tilde{u}_{n}(0)=0$, we obtain

$$
x^{\prime} \cdot \tilde{v}-C_{0} r^{2} \leqslant \tilde{u}(x) \leqslant x^{\prime} \cdot \tilde{v}+C_{0} r^{2} \quad \text { in } B_{r} \cap\left\{x_{n} \geqslant 0\right\},
$$


with $\tilde{v}_{i}=\tilde{u}_{i}(0), i=1, \ldots, n-1,|\tilde{v}| \leqslant \tilde{C}, \tilde{C}$ a universal constant. Therefore, for $k$ large enough we get

$$
x^{\prime} \cdot \tilde{v}-C_{1} r^{2} \leqslant \tilde{u}_{k}(x) \leqslant x^{\prime} \cdot \tilde{v}+C_{1} r^{2} \quad \text { in } \Omega_{r}\left(u_{k}\right)
$$

From the definition of $\tilde{u}_{k}$ the inequality above reads

$$
\varepsilon_{k} x^{\prime} \cdot \tilde{v}+x_{n}-\varepsilon_{k} C_{1} r^{2} \leqslant u_{k} \leqslant \varepsilon_{k} x^{\prime} \cdot \tilde{v}+x_{n}+\varepsilon_{k} C_{1} r^{2} \quad \text { in } \Omega_{r}\left(u_{k}\right)
$$

Set

$$
v=\frac{\left(\varepsilon_{k} \tilde{\nu}, 1\right)}{\sqrt{\varepsilon_{k}^{2}+1}} .
$$

Since, for $k$ large, $1 \leqslant \sqrt{\varepsilon_{k}^{2}+1} \leqslant 1+\varepsilon_{k}^{2} / 2$, we deduce from 4.9 that

$$
x \cdot v-\varepsilon_{k}^{2} r / 2-C_{1} r^{2} \varepsilon_{k} \leqslant u_{k} \leqslant x \cdot v+\varepsilon_{k}^{2} r / 2+C_{1} r^{2} \varepsilon_{k} \quad \text { in } \Omega_{r}\left(u_{k}\right) .
$$

In particular, if $r_{0}$ is such that $C_{1} r_{0} \leqslant 1 / 4$ and also $k$ is large enough so that $\varepsilon_{k} \leqslant 1 / 2$ we obtain

$$
x \cdot v-\varepsilon_{k} r / 2 \leqslant u_{k} \leqslant x \cdot v+\varepsilon_{k} r / 2 \quad \text { in } \Omega_{r}\left(u_{k}\right),
$$

which together with 4.3 implies that

$$
\left(x \cdot v-\varepsilon_{k} r / 2\right)^{+} \leqslant u_{k} \leqslant\left(x \cdot v+\varepsilon_{k} r / 2\right)^{+} \quad \text { in } B_{r} .
$$

Thus the $u_{k}$ satisfy the conclusion of the lemma, and we have reached a contradiction.

\section{The proofs of Theorems 1.1 and 1.2}

In this section we finally present the proofs of our main theorems.

Proof of Theorem 1.1. Let $u$ be a viscosity solution to 1.1 in $B_{1}$ with $0 \in F(u), g(0)=1$ and $a_{i j}(0)=\delta_{i j}$. Consider the sequence of rescalings

$$
u_{k}(x):=\frac{u\left(\rho_{k} x\right)}{\rho_{k}}, \quad x \in B_{1}
$$

with $\rho_{k}=\bar{r}^{k}, k=0,1, \ldots$, for a fixed $\bar{r}$ such that

$$
\bar{r}^{\beta} \leqslant 1 / 4, \quad \bar{r} \leqslant r_{0},
$$

with $r_{0}$ the universal constant of Lemma 4.1 .

Each $u_{k}$ solves (1.1) in $B_{1}$ with coefficients $a_{i j}^{k}(x)=a_{i j}\left(\rho_{k} x\right)$, right hand side $f_{k}(x):=$ $\rho_{k} f\left(\rho_{k} x\right)$, and free boundary condition $g_{k}(x):=g\left(\rho_{k} x\right)$. For the chosen $\bar{r}$, by taking $\bar{\varepsilon}=\varepsilon_{0}(\bar{r})^{2}$ the assumption (3.2) holds for $\varepsilon=\varepsilon_{k}:=2^{-k} \varepsilon_{0}(\bar{r})$. Indeed, in $B_{1}$, in view of (1.3),

$$
\begin{aligned}
\left|f_{k}(x)\right| & \leqslant\|f\|_{L^{\infty} \rho_{k}} \leqslant \bar{\varepsilon} \bar{r}^{k} \leqslant \varepsilon_{k}^{2}, \\
\left|g_{k}(x)-1\right| & =\left|g\left(\rho_{k} x\right)-g(0)\right| \leqslant[g]_{0, \beta} \rho_{k}^{\beta} \leqslant \bar{\varepsilon} \bar{r}^{k \beta} \leqslant \varepsilon_{k}^{2}, \\
\left|a_{i j}^{k}(x)-\delta_{i j}\right| & =\left|a_{i j}\left(\rho_{k} x\right)-a_{i j}(0)\right| \leqslant\left[a_{i j}\right]_{0, \beta} \rho_{k}^{\beta} \leqslant \bar{\varepsilon} \bar{r}^{k \beta} \leqslant \varepsilon_{k} .
\end{aligned}
$$


The hypothesis (1.2) guarantees that for $k=0$ also the flatness assumption 4.1) in Lemma 4.1 is satisfied by $u_{0}$. Then it easily follows by induction on $k$ and Lemma 4.1 that each $u_{k}$ is $\varepsilon_{k}$-flat in $B_{1}$ in the sense of (4.1). Now, a standard iteration argument gives the desired statement.

Proof of Theorem 1.2 Let $u$ be a viscosity solution to 1.1$]$ with $0 \in F(u)$ and $g(0)>0$. Without loss of generality, assume $g(0)=1$. Also, for simplicity we take $a_{i j}(0)=\delta_{i j}$.

Consider the blow-up sequence

$$
u_{k}:=u_{\delta_{k}}(x)=u\left(\delta_{k} x\right) / \delta_{k},
$$

with $\delta_{k} \rightarrow 0$ as $k \rightarrow \infty$. As in the previous theorem, each $u_{k}$ solves (1.1) with coefficients $a_{i j}^{k}(x)=$ $a_{i j}\left(\delta_{k} x\right)$, right hand side $f_{k}(x):=\delta_{k} f\left(\delta_{k} x\right)$, and free boundary condition $g_{k}(x):=g\left(\delta_{k} x\right)$. For $k$ large, the assumption (1.3) is satisfied for the universal constant $\bar{\varepsilon}$. In fact, in $B_{1}$,

$$
\begin{aligned}
\left|f_{k}(x)\right| & =\delta_{k}\left|f\left(\delta_{k} x\right)\right| \leqslant \delta_{k}\|f\|_{L^{\infty}} \leqslant \bar{\varepsilon} \\
\left|g_{k}(x)-1\right| & =\left|g_{k}(x)-g(0)\right| \leqslant\left[g_{k}\right]_{0, \beta}=\delta_{k}^{\beta}[g]_{0, \beta} \leqslant \bar{\varepsilon}, \\
\left|a_{i j}^{k}(x)-\delta_{i j}\right| & =\left|a_{i j}\left(\delta_{k} x\right)-a_{i j}(0)\right| \leqslant\left[a_{i j}\left(\delta_{k} x\right)\right]_{0, \beta}=\delta_{k}^{\beta}\left[a_{i j}\right]_{0, \beta} \leqslant \bar{\varepsilon} .
\end{aligned}
$$

Thus, using nondegeneracy and uniform Lipschitz continuity of the $u_{k}$ 's (see Appendix for a proof of these properties), standard arguments (see for example $[\overline{\mathrm{AC}}]$ ) imply that (up to extracting a subsequence):

(i) $u_{k} \rightarrow u_{0}<1$,

(ii) $\partial\left\{u_{k}>0\right\} \rightarrow \partial\left\{u_{0}>0\right\}$ locally in the Hausdorff distance,

for a globally defined function $u_{0}: \mathbb{R}^{n} \rightarrow \mathbb{R}$. The blow-up limit $u_{0}$ is a global solution to the free boundary problem

$$
\begin{cases}\Delta u_{0}=0 & \text { in }\left\{u_{0}>0\right\} \\ \left|\nabla u_{0}\right|=1 & \text { on } F\left(u_{0}\right)\end{cases}
$$

and since $F(u)$ is a Lipschitz graph in a neighborhood of 0 we also see from (i)-(ii) that $F\left(u_{0}\right)$ is Lipschitz continuous. Thus, it follows from [C1] that $u_{0}$ is a so-called one-plane solution, i.e. (up to rotations) $u_{0}=x_{n}^{+}$. Combining the facts above, one concludes that for all $k$ large enough, $u_{k}$ is $\bar{\varepsilon}$-flat say in $B_{1}$, i.e.

$$
\left(x_{n}-\bar{\varepsilon}\right)^{+} \leqslant u_{k}(x) \leqslant\left(x_{n}+\bar{\varepsilon}\right)^{+}, \quad x \in B_{1} .
$$

Thus $u_{k}$ satisfies the assumptions of Theorem 1.1 and our conclusion follows.

\section{Appendix}

We sketch here the proof of a standard result, that is, Lipschitz continuity and nondegeneracy of a solution $u$ to 3.1 3.2 .

Lemma 6.1 Let $u$ be a solution to 3.1p- 3.2) with $\varepsilon \leqslant \tilde{\varepsilon}$ a universal constant. If $F(u) \cap B_{1} \neq \emptyset$ and $F(u)$ is a Lipschitz graph in $B_{2}$, then $u$ is Lipschitz and nondegenerate in $B_{1}^{+}(u)$, i.e.

$$
c_{0} d(z) \leqslant u(z) \leqslant C_{0} d(z) \quad \text { for all } z \in B_{1}^{+}(u),
$$

with $d(z)=\operatorname{dist}(z, F(u))$ and $c_{0}, C_{0}$ universal constants. 
Proof. Assume without loss of generality that $0 \in B_{1}^{+}(u)$ and set $d:=d(0)$.

Consider the rescaled function

$$
\tilde{u}(x)=u(d x) / d, \quad x \in B_{1} .
$$

Clearly $\tilde{u}$ still satisfies 1.1 in $B_{1}$ with coefficients $\tilde{a}_{i j}(x)=a_{i j}(d x)$, right hand side $\tilde{f}(x)=$ $d f(d x)$ and free boundary condition $\tilde{g}(x)=g(d x)$. Since $d \leqslant 1$, the assumption 3.2 holds. We wish to show that

$$
c_{0} \leqslant \tilde{u}(0) \leqslant C_{0} .
$$

Assume for contradiction that $\tilde{u}(0)>C_{0}$, with $C_{0}$ to be made precise later.

To construct a subsolution, we use the same function as in Lemma 3.3 Precisely, let

$$
G(x)=C\left(|x|^{-\gamma}-1\right)
$$

be defined on the closure of the annulus $B_{1} \backslash \bar{B}_{1 / 2}$. In view of the uniform ellipticity of the coefficients, we can choose $\gamma$ large universal so that (for $\varepsilon$ small)

$$
\sum_{i j} \tilde{a}_{i j} G_{i j}>\varepsilon^{2} \quad \text { on } B_{1} \backslash \bar{B}_{1 / 2}
$$

and we can choose $C$ so that

$$
G=1 \quad \text { on } \partial B_{1 / 2} \text {. }
$$

By the Harnack inequality (see (3.9)), using the contradiction hypothesis we get (for $\varepsilon$ small)

$$
\tilde{u} \geqslant c \tilde{u}(0) \quad \text { on } \bar{B}_{1 / 2} \text {. }
$$

Thus, by the maximum principle,

$$
\tilde{u}(x) \geqslant v(x)=c \tilde{u}(0) G(x) \quad \text { on } \bar{B}_{1} \backslash B_{1 / 2} .
$$

Hence at the point $z$ where $d(0)$ is achieved we have

$$
|\nabla v|(z) \leqslant g(z) \leqslant 1+\varepsilon^{2} \leqslant 2,
$$

which contradicts $\tilde{u}(0)>C_{0}$ if $C_{0}$ is large enough.

To prove the lower bound, let

$$
\tilde{G}(x)=\eta(1-G(x))
$$

with $\eta$ (depending on $\gamma$ ) such that

$$
|\nabla \tilde{G}|<1-\varepsilon^{2} \quad \text { on } \partial B_{1 / 2} \text {. }
$$

Assume without loss of generality that $F(u)$ is a Lipschitz graph in the $x_{n}$ direction with Lipschitz constant equal to 1 . We translate the graph of $\tilde{G}$ by $-4 e_{n}$. Notice that it is above the graph of $\tilde{u}$ since $\tilde{u} \equiv 0$ in $B_{1}\left(-4 e_{n}\right)$. We slide the graph of $\tilde{G}$ in the $e_{n}$ direction till we touch the graph of $\tilde{u}$. Since $\tilde{G}$ is a strict supersolution to our free boundary problem, the touching point $\tilde{z}$ can occur only on the $\eta$ level set with $\tilde{d}:=d(\tilde{z}, F(u)) \leqslant 1$. From the first part, $\tilde{u}$ is Lipschitz continuous and hence $\tilde{u}(\tilde{z})=\eta \leqslant C \tilde{d}$. Thus

$$
C^{-1} \eta \leqslant \tilde{d} \leqslant 1
$$


that is, $\tilde{d}$ is comparable to 1 . Since $F(u)$ is Lipschitz we can connect 0 and $\tilde{z}$ with a chain of intersecting balls included in the positive side of $\tilde{u}$ with radii comparable to 1 . The number of balls is bounded by a universal constant. Then we can apply the Harnack inequality to obtain (for $\varepsilon$ small)

$$
\tilde{u}(0) \geqslant c \tilde{u}(\tilde{z})=c_{0},
$$

as desired.

\section{REFERENCES}

[AC] Alt, H. W., \& CAFFARElli, L. A. Existence and regularity for a minimum problem with free boundary. J. Reine Angew. Math. 325 (1981), 105-144. Zbl 0449.35105 MR 0618549

[AFT] Amick, C. J., Fraenkel, L. E., \& Toland, J. F. On the Stokes conjecture for the wave of extreme form. Acta Math. 148 (1982), 193-214. Zbl 0495.76021 MR 0666110

[AF] ARgiolas, R., \& FERRARI, F. Flat free boundaries regularity in two-phase problems for a class of fully nonlinear elliptic operators with variable coefficients. Interfaces Free Bound. 11 (2009), 177-199. Zbl 1179.35349 MR 2511639

[C1] CAfFarelli, L. A. A Harnack inequality approach to the regularity of free boundaries. Part I: Lipschitz free boundaries are $C^{1, \alpha}$. Rev. Mat. Iberoamer. 3 (1987), 139-162. Zbl 0676.35085 MR 0990856

[C2] CAfFarelli, L. A. A Harnack inequality approach to the regularity of free boundaries. Part II: Flat free boundaries are Lipschitz. Comm. Pure Appl. Math. 42 (1989), 55-78. Zbl 0676.35086 MR 0973745

[CC] Caffarelli, L. A., \& Cabré, X. Fully Nonlinear Elliptic Equations. Amer. Math. Soc. Colloq. Publ. 43, Amer. Math. Soc., Providence, RI (1995). Zbl 0834.35002 MR 1351007

[CFS] Cerutti, M. C., Ferrari, F., \& Salsa S. Two phase problems for linear elliptic operators with variable coefficients: Lipschitz free boundaries are $C^{1, \gamma}$. Arch. Ration. Mech. Anal. 171 (2004), 329348. Zbl 1106.35144 MR 2038343

[CS] Constantin, A., \& Strauss, W. Exact steady periodic water waves with vorticity. Comm. Pure Appl. Math. 57 (2004), 481-527. Zbl 1038.76011 MR 2027299

[F1] FELdman, M. Regularity of Lipschitz free boundaries in two-phase problems for fully nonlinear elliptic equations. Indiana Univ. Math. J. 50 (2001), 1171-1200. Zbl 1037.35104 MR 1871352

[F2] Feldman, M. Regularity for nonisotropic two-phase problems with Lipshitz free boundaries. Differential Integral Equations 10 (1997), 1171-1179. Zbl 0940.35047 $\mid$ MR 1608061

[Fe] FERraRI, F. Two-phase problems for a class of fully nonlinear elliptic operators, Lipschitz free boundaries are $C^{1, \gamma}$. Amer. J. Math. 128 (2006), 541-571. Zbl 1142.35108 MR 2230916

[FS1] FERRARI, F., \& SALSA, S. Regularity of the free boundary in two-phase problems for elliptic operators. Adv. Math. 214 (2007), 288-322. Zbl 1189.35385 MR 2348032

[FS2] Ferrari, F., \& SAlsa, S. Subsolutions of elliptic operators in divergence form and application to two-phase free boundary problems. Bound. Value Probl. 2007, art. ID 57049, 21 pp. Zbl 1188.35070 MR 2291927

[KN] Kinderlehrer, D., \& Nirenberg, L. Analyticity at the boundary of solutions of nonlinear second-order parabolic equations. Comm. Pure Appl. Math. 31 (1978), 283-338. Zbl 0391.35045 MR 0460897

[P] Plotnikov, P. I. Proof of the Stokes conjecture in the theory of surface waves. Dinamika Splosh. Sredy 57 (1982), 41-76 (in Russian); English transl.: Stud. Appl. Math. 3 (2002), 217-244. Zbl 1152.76339 MR 1883094

[S] SAVIN, O. Small perturbation solutions for elliptic equations. Comm. Partial Differential Equations 32 (2007), 557-578. Zbl pre05174527 MR 2334822 
[V] VARVARUCA, E. On the existence of extreme waves and the Stokes conjecture with vorticity. J. Differential Equations 246 (2009), 4043-4076. Zbl 1162.76011 MR 2514735

[VW] Varvaruca, E., \& Weiss, G. S. A geometric approach to generalized Stokes conjectures. Preprint 2009, arXiv:0908.1031.

[W1] WANG, P. Y. Regularity of free boundaries of two-phase problems for fully nonlinear elliptic equations of second order. I. Lipschitz free boundaries are $C^{1, \alpha}$. Comm. Pure Appl. Math. 53 (2000), 799-810. Zbl 1040.35158 MR 1752439

[W2] WANG, P. Y. Regularity of free boundaries of two-phase problems for fully nonlinear elliptic equations of second order. II. Flat free boundaries are Lipschitz. Comm. Partial Differential Equations 27 (2002), 1497-1514. Zbl 1125.35424 MR 1924475 